\title{
Efectividad de un programa de promoción de la salud en la disminución de costos médicos asistenciales
}

\author{
A health promotion programme's effectiveness in reducing medical \\ care costs
}

Elkin Martínez-López e Isabel C. Grajales

Facultad Nacional de Salud Pública, Universidad de Antioquia. Medellín, Colombia. elkmartz@guajiros.udea.edu.co, leisagra@guajiros.udea.edu.co

Recibido 28 Junio 2010/Enviado para Modificación 7 Noviembre 2010/Aceptado 2 Diciembre 2010

RESUMEN

Objetivo La práctica regular de actividad física sería capaz de reducir la frecuencia de enfermedades y podría repercutir en la reducción de costos médicos asistenciales. Explorar la posible reducción de costos asistenciales en personas activas.

Métodos En una institución prestadora de servicios de salud, se contrastan los costos médicos de un grupo de personas activas con un grupo control de personas tomadas aleatoriamente del resto de la población afiliada.

Resultados Los costos médicos son más bajos en las personas activas en aspectos como urgencias, hospitalizaciones y medicamentos. Se observa reducción en la frecuencia de los casos y en el costo promedio por paciente y por servicio.

Conclusión La actividad física practicada en forma regular reduce los requerimientos de atención médica y con ello se genera un ahorro significativo en los costos asistenciales. Se abren perspectivas promisorias para la implementación de programas de promoción de la salud a nivel poblacional y para la racionalización de los recursos económicos en el sector salud.

Palabras Clave: Servicios de salud, costos y análisis de costo, promoción de la salud, actividad motora (fuente: DeCS, BIREME).

\section{ABSTRACT}

Objective Regularly engaging in physical activity should enable disease incidence to become reduced and may result in reducing healthcare costs. Exploring possible health care cost reduction in active people.

Methods An active group of people's medical costs were contrasted with those of a control group of people taken at random from the rest of the population so covered in a health-care providing institution.

Results Medical costs were lower for active people in areas such as emergency room visits, hospitalization and providing medication; a reduction was observed in the frequency of cases and the average cost per patient and per service.

Conclusion Regular physical activity reduces health care requirements and thereby 
leads to significant savings in health-care costs. This leads to promising perspectives for implementing health promotion programmes amongst the population and rationalising health sector financial resources.

Key Words: Health service, costs and cost analysis, health promotion, motor activity (source: MeSH, NLM).

a inactividad física es un grave problema de salud pública y tiene serias repercusiones también para la economía familiar y comunitaria (1-4). Tres de cada cuatro personas en el mundo no realizan actividad física suficiente para proteger su salud y esta realidad se extiende también a Colombia y América Latina $(5,6)$.

La inactividad física se asocia con enfermedades cardiovasculares, infarto del miocardio, enfermedad cerebrovascular, hipertensión, cáncer de colon, cáncer de mama, diabetes, osteoporosis, depresión, ansiedad, y otros efectos desfavorables para la salud $(7,8)$.

La inactividad física también se asocia con altos costos en prestación de servicios de salud. Los estudios reportan valores que oscilan entre 3 y $35 \%$ de los costos directos en servicios médicos asistenciales relacionados con enfermedades asociadas al sedentarismo y la obesidad $(9,10)$.

Los economistas han llamado la atención de los gobiernos para analizar la inversión en salud, pues se ha establecido que muchas de las acciones y de las intervenciones que se realizan en el sector son de alto costo y de baja efectividad. Recomiendan mirar hacia la prevención y la promoción de la salud, si no por razones epidemiológicas, al menos por razones económicas, dado que los costos de las enfermedades de alta complejidad técnica asociados con el sedentarismo son prácticamente insostenibles $(11,12)$.

Es sensato pensar que si la inactividad física tiene tan graves repercusiones en la salud y la economía de los países, sería conveniente promover la actividad física como una estrategia inteligente de promoción de la salud y del bienestar social.

En Colombia, se desconoce el impacto que tiene la inactividad física en la salud pública y en los altos costos del sistema de prestación de servicios de salud y es todavía más escaso encontrar estudios que exploren los beneficios 
de la actividad física en los diversos campos de la salud y en la reducción de costos médicos asistenciales.

La pregunta fundamental que nos hacemos en el presente estudio se refiere a averiguar si realmente la actividad física produce alguna disminución en los costos asistenciales y médicos para quienes practican el ejercicio en forma regular y recreativa. Confiamos en que los resultados aportarán luz para la implementación de programas de promoción de la salud que sean a la vez beneficiosos para la salud pública y para las economías familiar, institucional y comunitaria.

\section{MÉTODOS}

Tipo de estudio

Diseño analítico que contrasta los costos generados por la demanda de servicios de salud en personas activas y en personas controles, pertenecientes todas ellas a la comunidad afiliada al programa de seguridad social en salud implementado en una institución de educación superior.

Población y muestra

La población está conformada por todas las personas afiliadas al programa de salud de la Universidad de Antioquia durante el año 2003. El diseño epidemiológico para explorar la influencia del sedentarismo parte de la conformación de dos grupos, así:

Activos. Conformado por 191 sujetos (95 mujeres y 96 hombres) pertenecientes al programa para la salud sico-física (Prosa) que ofrece el departamento de bienestar universitario de la Universidad de Antioquia a sus empleados. Los participantes realizan 4-5 sesiones semanales de actividad física moderada, cada sesión dura cerca de 60 minutos, se hace énfasis en la recreación y se complementa con diversas conferencias sobre estilos de vida saludable. Se definió como persona activa a quien registra una participación regular en el programa de mantenimiento físico, superior a un año.

Grupo control. Conformado por 382 sujetos seleccionados de la base de datos de afiliados al programa de salud de la Universidad. Para lograr una buena comparabilidad con el grupo de activos, se hizo selección aleatoria para cada sexo por aparte y en cada grupo etáreo para alcanzar una proporción de 2 controles por cada individuo activo y obtener una muestra que fuera a 
la vez representativa de la comunidad afiliada y comparable con el grupo participante del programa para la salud sicofísica

Procedimientos

La información de la población afiliada fue suministrada directamente por el Sistema de Seguridad Social en salud de la Universidad de Antioquia, el cual incluye tanto el proceso de aseguramiento como la prestación de servicios de salud. Las actividades asistenciales se realizan a través de la IPS Universitaria, donde se consigna cada procedimiento con alto nivel de confiabilidad dado que los procesos de registro están gerenciados por profesionales en sistemas de información en salud calificados y expertos en este tipo de manejo informático.

Se revisan las facturas por servicios de salud en todos los afiliados durante el año 2003. Esta información se condensa en los registros individuales de prestación de servicios y contiene los costos por actividades de diagnóstico, procedimientos, medicamentos, hospitalizaciones, urgencias y en general todos los costos que se aplican a la atención del paciente. Las bases de datos se revisan cuidadosamente para asegurar la más alta precisión.

Manejo y análisis de las bases de datos

Inicialmente se revisan las estructuras de los archivos de datos y se pasan a un formato único (archivos de Excel), posteriormente se organizan en una base de datos de Microsoft Access. Una vez identificados los grupos de personas a estudiar (activos y grupo control) se procedió al cruce con las base de datos de prestación de servicios, esto con el fin de identificar la demanda y costos de los servicios para cada grupo. Esta información fue procesada en repetidas ocasiones para verificar la consistencia de los resultados.

Variables

Nuestro interés se centró en evaluar distintos servicios asistenciales, especialmente aquellos donde los costos directos hacían posible conocer la cuantificación más precisa y que a su vez representaban rubros importantes de la factura médica, tales como, emergencias médicas, hospitalizaciones, y medicamentos. Para cada uno de estos servicios fue posible conocer los gastos totales, los requerimientos por paciente y el costo medio del servicio.

Análisis estadístico

El análisis univariado estudia la distribución de frecuencias para establecer 
estimadores de posición y de dispersión y para explorar los criterios de normalidad a fin de utilizar pruebas paramétricas cuando ello es posible. Las pruebas de hipótesis se establecen sobre las diferencias entre el grupo de personas activas y las personas del grupo control. Se prueban diferencias de promedios con el referente estadístico del análisis de varianza y también se recurre a las comparaciones de medianas a través de las pruebas no paramétricas tipo Kruskal-Wallis. Las diferencias en proporciones se prueban con el estadístico $\mathrm{X}^{2}$ de independencia.

\section{RESULTADOS}

La población afiliada al programa de salud de la Universidad de Antioquia en el año 2003, está conformada por 8347 personas. La variación en edad de los afiliados es muy amplia, abarca desde niños de un año hasta adultos mayores octogenarios, aunque la mayor proporción de afiliados oscila entre 35 y 70 años con un leve predominio de los hombres (55\%) sobre las mujeres $(45 \%)$.

Durante el año, la IPS prestó servicios a sus afiliados por un valor de 4 730 millones de pesos, el $83 \%$ directamente y el $17 \%$ restante mediante la contratación con terceros. Los servicios que generaron mayores costos fueron procedimientos, hospitalización y medicamentos.

El grupo de 191 personas activas, durante este mismo año, demandó servicios por 15,5 millones de pesos en urgencias, hospitalizaciones y medicamentos. El grupo control (382 personas) por su parte generó costos por 49,9 millones por el mismo concepto.

Al realizar un análisis más detallado de cada uno de los servicios ofrecidos por la IPS a sus usuarios, encontramos diferencias en la demanda de los servicios de estos dos grupos (activos y controles) en cada uno de los servicios de urgencias, medicamentos y hospitalización (Tabla 1).

Los activos demandaron 1,3 millones de pesos al año por atenciones de urgencias, los controles por su parte requirieron atenciones en este mismo servicio por un total de 11,9 millones. Un activo cuesta en promedio $\$ 6640$ pesos/año en el rubro de emergencias medicas, un control \$ 31253 pesos/ año, lo que representa un costo 4,6 veces mayor en este grupo con relación a los activos. 
Tabla 1. Comparación de costos por urgencias, hospitalización y medicamentos en activos y en controles

\begin{tabular}{llccc}
\hline Servicio & Activos & $\begin{array}{c}\text { Frecuencia } \\
\text { (por } 100 \\
\text { personas/año) }\end{array}$ & $\begin{array}{c}\text { costo medio } \\
\text { servicio } \\
\text { (miles) }\end{array}$ & $\begin{array}{c}\text { costo medio } \\
\text { persona } \\
\text { (miles) }\end{array}$ \\
\hline Urgencias & Controles & 3,14 & 211,5 & 6,64 \\
Hospitalización & Activos & 7,52 & 426,4 & 31,25 \\
& Controles & 4,76 & $730 *$ & 61,48 \\
Medicamentos & Activos & 133,5 & $1401{ }^{*}$ & 73,05 \\
& Controles & 277,5 & 9,92 & 13,24 \\
${ }^{*}$ Medianas & & & 9,38 & 26,04 \\
\hline
\end{tabular}

La Hospitalización generó costos anuales para el grupo de los activos por 11,7 millones y para los controles por 28 millones. Un activo cuesta en promedio 61487 pesos/año, mientras que un control 73050 pesos/año, lo cual representa un exceso promedio en el costo de $20 \%$ para los controles en relación con los activos.

En cuanto a la demanda de medicamentos se observó que los activos requirieron prescripciones por 2,5 millones de pesos/año, de otro lado el grupo control requirió medicamentos por un total de 10 millones. En promedio, un activo al año consume medicamentos por 13244 pesos, un control consume $\$ 26038$, lo que representa el doble del costo para los controles con relación a los activos.

En los servicios de consultas especializadas y procedimientos, se encontró que el costo promedio de atenciones demandadas por los activos fue muy similar al del grupo control (\$129 mil pesos/año los activos y 124 mil pesos/año controles)

\section{Urgencias}

En los activos se presentaron 6 urgencias que ascendieron a un costo de 1,27 millones de pesos, en los controles se presentaron 28 urgencias que costaron 11,9 millones.

La prevalencia de urgencias en los activos (3,14/100 personas/ año) fue $57 \%$ más baja que en los controles (7,33) (RR 2,33 X2 5,2 p ,02) (Figura 1).

El costo medio por urgencia en los activos $(\$ 211.504)$ fue $50.5 \%$ más bajo que el costo medio en los controles (\$ 426376 ) (F 179 p .00).

El costo de la factura total por urgencias en los afiliados, ascendió en el año a 254,6 millones de pesos, por lo tanto, podría presumirse que si todos los afiliados 
fueran activos se necesitaría un gasto solamente de 55,4 millones, lo cual implicaría un ahorro potencial de 199,2 millones de pesos, lo que a su vez significaría una reducción del 78,2 \% en la factura total por urgencias.

Figura 1. Frecuencia de casos por urgencias en controles y activos

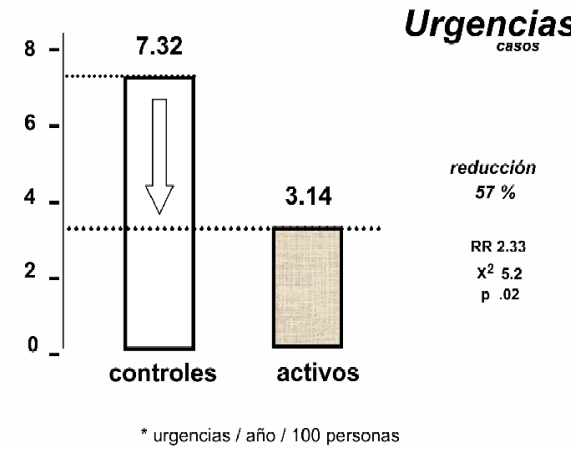

Hospitalizaciones

Durante el periodo, se presentaron 7 hospitalizaciones en el grupo de los activos que representaron un costo de 11,7 millones de pesos. En los controles ocurrieron 18 hospitalizaciones que tuvieron un costo de 27,9 millones.

La frecuencia de hospitalizaciones en los activos: 3,66/100 personas/año, fue $22,3 \%$ más baja que la frecuencia en el grupo control: 4,71 (RR 1,29)

La mediana del costo por hospitalización en los activos fue de \$ 729 953, lo cual representa un valor $47,9 \%$ más bajo que la mediana del costo por hospitalización en los controles \$1 401158 ( KW 2.6 p .10 ). (Figura 2)

El costo de la factura total por hospitalizaciones fue de 909,3 millones en el año, por lo tanto, cabe presumir que si todos los afiliados fueran activos el costo esperado por este concepto habría sido de 236,8 millones, lo cual implicaría un ahorro potencial de 672,2 millones, lo que a su vez significaría una reducción del $73,9 \%$ en la factura total por hospitalizaciones.

\section{Medicamentos}

En los activos se presentaron 255 prescripciones con un costo de 2,53 millones de pesos, en los controles se presentaron 1060 prescripciones con un costo de 9,95 millones. 
La frecuencia de facturas por medicamentos en los activos (133,5/100 personas /año) es $52 \%$ más baja que en los controles $(277,5)$.

El costo medio por prescripción en los activos fue de $\$ 9919$, valor muy similar al costo medio en los controles \$9384.

El costo promedio de la medicación por persona activa es de \$ 13244 valor que es un 49,1\% más bajo que el costo promedio de la medicación en las personas controles (\$ 26 038) (Figura 3).

Figura 2. Costo medio de hospitalización en controles y activos

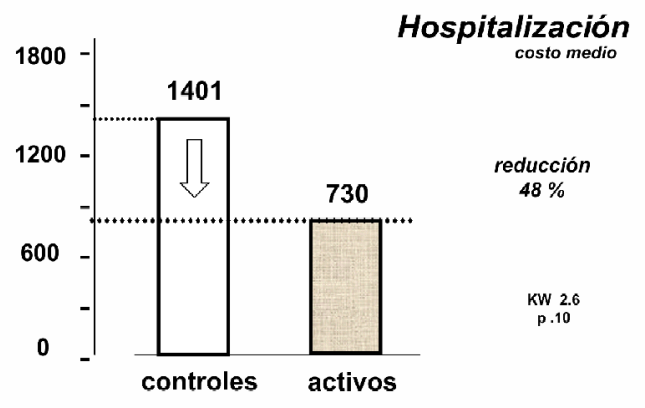

* mediana del costo en miles de pesos

Figura 3. Costo medio de medicamentos por persona en controles y activos

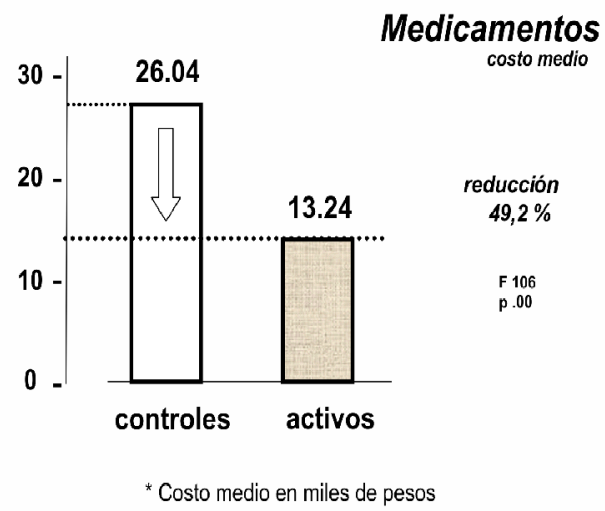


El costo de la factura total por medicamentos durante el año fue de 625 millones de pesos. Si todos los afiliados hubieran sido activos los requerimientos por medicación hubieran ascendido a 110,5 millones, lo cual hubiera representado un ahorro de 514,5 millones, lo que a su vez significaría una reducción del $82,2 \%$ en la factura total por medicamentos.

\section{DISCUSIÓN}

Efectivamente las personas activas registran una reducción significativa en el número de consultas por urgencias superior en este caso al $50 \%$, lo cual estaría hablando de mejores condiciones de salud de los activos en comparación con los demás afiliados, pero además los costos promedios de dichas demandas de servicio fueron más bajos que los controles, lo cual a su vez estaría diciendo que la gravedad de los hechos también pudo haber sido menor. Estos datos concuerdan con otros estudios que reportan reducciones del $40-50 \%$ en consultas de urgencias y estancias hospitalarias para adultos que caminan regularmente (13).

En las hospitalizaciones pudo observarse una diferencia similar, que no solo implicó una reducción en la frecuencia de los ingresos hospitalarios, sino que además implicó una reducción en los costos medios por hospitalización, indicando con ello que las resolución de las necesidades debió haber sido más simple y corta en los activos. Estos hallazgos concuerdan con otro estudio realizado en funcionarios de una institución pública, donde se pudo establecer que las incapacidades por causas médicas son menores en las personas activas tanto en frecuencia como en duración (14).

En los medicamentos también es aparente una reducción en el número de prescripciones y la vez el costo medio de los medicamentos requeridos fue más bajo en los activos que los controles. Todo esto apunta en forma consistente a reconocer que realmente las personas activas reducen los requerimientos de atención médica y a su vez reducen los costos derivados de todos los procesos terapéuticos.

¿Sobre qué bases puede argumentarse que las personas activas pudieran estar favorecidas con un mejor nivel de salud y por ende con una reducción de sus demandas para la atención médica? Podría decirse que las evidencias a favor de los efectos beneficiosos de la actividad física para la salud resultan hoy en día bastante sólidas y consistentes. 
La actividad física regular aumenta la salud cardiovascular y previene la aparición de la enfermedad isquémica del corazón y del cerebro, la insuficiencia cardíaca y otras manifestaciones periféricas de la arterioesclerosis. La hipertensión arterial se controla en buena medida con la reducción de peso corporal, sobre todo cuando ella se obtiene a través de la práctica de ejercicio físico regular. Dado que las enfermedades cardiovasculares son la principal causa de enfermedad, incapacidad y muerte en los adultos, cabe esperar que la actividad física muestre su impacto positivo en la carga que estas afecciones causan al monto global de la morbilidad y de hecho también a los costos que ellas generan $(15,16)$.

La actividad física tiene también notables efectos sobre la reducción de la obesidad, de la diabetes, de ciertos tipos de cáncer como el de colon y el de mama, lo cual representa un favorable potencial de evitación para las enfermedades que conocemos como "de alto costo" por su complejidad clínica, por su cronicidad y por la alta demanda de servicios y medicamentos $(17,18)$. Hacemos referencia a las enfermedades cardiovasculares, al cáncer en todas sus variedades, y a las nefropatías severas que requieren procesos costosos de hemodiálisis (19).

Diversos estudios en el mundo han empezado a mostrar el impacto positivo de la práctica de la actividad física regular en la reducción de los costos por asistencia médica (20-26). De hecho, las inversiones que se hacen para construir y dotar técnicamente los escenarios para la práctica de actividad física se ven bien recompensadas con los ahorros que se logran en las reducciones de las facturas por gastos médicos (27).

Incluso con actividades tan simples como caminar regularmente se obtienen reducciones del 40-60 \% en requerimientos de atención médica $(13,28)$. La actividad física regular permitiría una considerable reducción en los costos directos por servicios médicos asistenciales, sin hablar de los beneficios indirectos que son aún mayores cuando se considera la ganancia en productividad por la reducción de incapacidades y sobre todo por la posibilidad de disminuir la frecuencia de muertes prematuras (3).

Las limitaciones del presente estudio se refieren por lo pronto al corto período de observación y a la incipiente cultura de registrar y codificar todos los procesos de facturación y consolidación de bases unificadas por la identificación de los costos que genera cada usuario. Con el desarrollo de la sistematización de las bases de datos en las instituciones aseguradoras y de 
prestación de servicios, estas evidencias podrán constatarse progresivamente, incluyendo también buena parte de los costos indirectos que hoy en día se subregistran y que corresponden a una gran proporción de la alta carga que el sedentarismo genera en la comunidad.

Apoyados en el desarrollo de la tecnología electrónica, el eslogan de la vida moderna parece ser que todo se puede realizar con solo mover un dedo, de hecho esa es toda la actividad física que mucha gente realiza en el transcurso de un día. El sedentarismo se ha convertido en un rasgo de la era tecnológica, pero sus consecuencias desfavorables en la salud pública y en el balance económico de las comunidades contemporáneas, obliga a plantear planes y programas para contrarrestar esta inercia masiva y creciente en la población $(29,30)$.

La actividad física ha de ser una de las estrategias fundamentales en la promoción de la salud del presente y el futuro, pues su impacto sobre el mejoramiento del bienestar de la salud es ahora prácticamente incuestionable, además de los amplios beneficios que se consiguen con el aprovechamiento más racional de los recursos que de por si son siempre escasos para tantas necesidades

Agradecimientos: Hacemos reconocimiento a la Institución Prestadora de Servicios de Salud-IPS Universitaria- de la Universidad de Antioquia, tanto en el nivel gerencial como en el nivel técnico, por proveer amablemente la información requerida para este estudio.

\section{REFERENCIAS}

1. Allender S, Foster C, Scarborough P, Rayner M. The burden of physical activity-related ill health in the UK report. J Epidemiol community health 2007; 61(4):344-348.

2. World Health Organization. Sedentary lifestyle a global public health problem. Geneva: World Health Organization; 2002.

3. Katzmarzyk P, Gledhill N, Shephard R. The economic burden of physical inactivity in Canada. CMAJ. 2000; 163(11): 1435-40.

4. Pratt M, Macera C, Wang G. Higher direct medical costs associated with physical inactivity. Physician sports med. 2000; 28: 63-70.

5. Martínez E, Saldarriaga J. Actividad Física y Estilo de Vida en Medellín. Un desafió para la Promoción de la Salud. Rev Fac Nac Salud Pública. 2008; 26 (2):117-123.

6. León MH, Martínez E. Factores de riesgo para enfermedades crónicas, Bucaramanga - SantanderColombia. Iniciativa CARMEN. Ed. Universidad de Antioquia; 2007.

7. Anderson L, Martison BC, Crain A, Pronk N, Whiterbird R, O"Connor P, Fine L. Health care charges associated with physical inactivity, overweight, and obesity. Prev Chronic Dis. 2005; 2(4):A09.

8. Wang F, McDonald T, Champagne LJ, Edington DW. Relationship of body mass index and physical activity to health care costs in employee. J Occup Environ Med. 2004;46(5):42836. 
9. Garret N, Brasure m, Schmitz K, Schultz M, Huber M. Physical Inactivity: Direct cost to a health plan. Am J of Prev Med. 2004; 27(4): 304-9.

10. Katzmarzyk P, Janssen I. The economic costs associated with physical inactivity and obesity in Canada. Un update. Canad J of Appl Physiol. 2004; 29 (1): 90-115.

11. Banco Mundial. Informe sobre el desarrollo mundial: Invertir en salud. Washington, D. C.; 1993.

12. Cunningham S. An introduction to economic evaluation of health care. British Orthodontic Society Londres. $2001 ; 28(3): 246-250$.

13. Perkins A, Clark D. Assessing the association of walking with health services use and costs among socioeconomically disadvantaged older adults. Prev med. 2001; 32(6): 492-501.

14. Martínez E, Saldarriaga J. Inactividad física y ausentismo en el ámbito Laboral. Rev. salud pública (Bogotá). 2008; 10(2): 227-238.

15. Wang G, Pratt M, Macera C, Zheng Z, Heath G. Physical activity, cardiovascular disease and medical expenditure in US adults. Ann Behav Med. 2004. Oct; 28(2): 88-94.

16. Wannamethee SG, Shaper AG. Physical activity in the prevention of cardiovascular disease: An epidemiological perspective. Sports Medicine. 2001; 34(2):101-114.

17. Stromme SB, Hostmark AT. Physical activity overweight and obesity. Sports Medicine. 2000; 120(2):578-582

18. Proper $\mathrm{KI}$, de Bruyne MC, Hildebrandt VH, van der Beek AJ, Meerding WJ, van Mechelen W. Costs, benefits and effectiveness of worksite physical activity counseling from the employer's perspective. Scand J Work Environ Health; 30(1): 36-46.

19. David IM, Medina AM, Martínez E. Enfermedades de alto costo en afiliados a un sistema institucional de aseguramiento y prestación de servicios de salud. Rev Fac Nal Salud Pública. 2006; 24(2): 98-104.

20. Wang F, McDonald T, Reffitt B, Edington DW. Physical activity, and health care utilization/costs among Medicare retirees. 2005;13(8):1450-7.

21. Ackermann RT, Cheadle A, Sandhu N, Madsen L, Wagner EH, LoGerfo JP. Community exercise program use and changes in healthcare costs for older adults. Am J Prev Med. 2003; 25(3): 232-7.

22. Martinson BC, Crain AL, Pronk NP, O'Connor PJ, Maciosek MV. Changes in physical activity and short-term changes in health care charges: a prospective cohort study of older adults. Prev Med. 2003; 37(4): 319-26.

23. Patrick DL, Ramsey SD, Spencer AC, Kinne S, Belza B, Topolski TD.Economic evaluation of aquatic exercise for persons with osteoarthritis. Med Care. 2001; 39(5): 413-24.

24. Elley R, Kerse N, Arroll B, Swinburn B, Ashton T, Robinson E. Cost-effectiveness of physical activity counseling in general practice. N Z Med J. 2004; 117(1207): U1216.

25. Van den Heuvel S, Boshuizen H, Hildebrandt V, Blatter B, Ariëns G, Bongers P. Effect of sporting activity on absenteeism in a working population. $\mathrm{Br} \mathrm{J}$ Sports Med. 2005; 39:1-5.

26. Schnelle JF, Kapur K, Alessi C, Osterweil D, Beck JG, Al-Samarrai NR, et al. Does an exercise and incontinence intervention save healthcare costs in a nursing home population? J Am Geriatr Soc. 2003;51(2):161-8.

27. Wang G, Macera C, Scudder-Soucie B, Schmid T, Pratt M, Buchner D. A cost-benefit analysis of physical activity using bike/ pedestrian trails. Health promot pract. 2005; 6 (2):174-9.

28. Tsuji I, Takahashi K, Nishino Y, Ohkubo T, Kuriyama S, Watanabe Y, et al. Impact of walking upon medical care expenditure in Japan: the Ohsaki Cohort Study. Int J Epidemiol. 2003: 32(5): 809-14.

29. US Dept Of Health-CDC. Promoting physical activity: A guide for community action. Champaing: Human Kinetics; 2002.

30. Pratt M, Jacoby E. Promoting physical activity in the Americas. Medicine Science and Sports Exercise. 2004; 25(2):183-190. 